\title{
Usos del aprendizaje-servicio durante el proceso de formación de estudiantes en Educación Física: una revisión sistemática
}

\author{
Alonso Peña, \\ Cristóbal Delgado \\ Universidad de Santiago de Chile, Chile
}

\section{Resumen}

¿Son pertinentes las experiencias de aprendizaje-servicio en la formación de futuros profesores de Educación Física? La presente investigación tiene como fin una revisión sistemática de las experiencias de aprendizaje-servicio realizadas en la formación profesional de pregrado de la carrera de educación física que colaborará en la respuesta a la pregunta inicial. La metodología se realizó bajo un diseño de corte exploratorio con un plan de revisión sistemática que contempla diversos criterios para la selección de los estudios, una estrategia de exploración y métodos para la recolección y síntesis de la información. El resultado fue de 774 artículos en la plataforma ERIC, de los que resultaron finalmente 8 publicaciones seleccionadas, las que fueron analizadas y se recogen sus métodos, resultados y conclusiones para la implementación curricular de proyectos de aprendizaje-servicio en la formación inicial docente del ámbito de la educación física dado el impacto el compromiso social y responsabilidad ciudadana en su desarrollo profesional.

\section{Palabras clave}

Aprendizaje-servicio, educación física, formación pregrado. 


\title{
The application of service-learning in the training of PE teachers: a systematic review
}

\begin{abstract}
Are service-learning experiences relevant in the training of future Physical Education teachers? In order to answer this question, this research aimed at conducting a systematic review of service-learning experiences carried out as part of the professional training of the Physical Education undergraduate degree. The methodology followed an exploratory design with a systematic review plan that included various criteria for the selection of studies, as well as an exploration strategy and methods for the collection and synthesis of information. The review included 774 articles from the ERIC platform, out of which 8 publications were selected for analysis, and their methods, results and conclusions were collected for the curricular implementation of service-learning projects in initial teacher training in the field of physical education, given the significance of social commitment and civic responsibility in their professional development.
\end{abstract}

\section{Keywords}

Service-learning, physical education, undergraduate training. 


\section{Introducción ${ }^{1}$}

El aprendizaje-servicio representa una oportunidad para reducir la brecha entre teoría y práctica en la formación de pregrado (Chiva-Bartoll et al., 2020). En este sentido, al hablar de aprendizaje-servicio, nos referimos a una experiencia educativa basada en un curso, con créditos, en la que los estudiantes; (a) participan en una actividad de servicio organizada que responde las necesidades identificadas en la comunidad; y (b) reflexionan sobre la actividad de servicio, de manera que se comprenda mejor el contenido del curso, se logre una apreciación más amplia de la disciplina y un mayor sentido de responsabilidad cívica (Bringle and Hatcher, 1995, como se citó en Chiva-Bartoll et al., 2020). Del mismo modo, Abal de Hevia (2016) reconoce la existencia de diversas definiciones del aprendizaje-servicio, identificando tres elementos propios del aprendizaje-servicio y que son compartidos por la gran mayoría de conceptualizaciones. En primer lugar, el protagonismo estudiantil en todas las fases del proyecto. En segundo lugar, la articulación de los aprendizajes curriculares con el servicio solidario. $Y$ por último, la organización de actividades solidarias sobre necesidades reales y sentidas por las comunidades. Con lo anterior, se valida la concepción de aprendizaje-servicio como sigue:

\footnotetext{
1 Se agradece a la Dirección de Pregrado de la Universidad de Santiago de Chile, la aprobación del Proyecto de Innovación Pedagógica:

"Intervenciones de Pedagogía en Educación Física bajo metodología de Aprendizaje-Servicio N०041/2018"
}

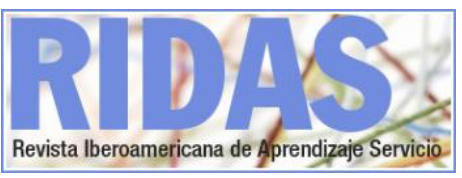

"Como práctica pedagógica, el aprendizaje-servicio es una modalidad de intervención que promueve actividades estudiantiles solidarias, no sólo para atender necesidades de la comunidad, sino para la mejora del aprendizaje académico y la formación personal en valores, estimulando la participación ciudadana responsable (Tapia, 2000, como se citó en Abal de Hevia, 2016). En esta concepción, los estudiantes aprenden y se desarrollan a través de la activa participación en un servicio solidario cuidadosamente organizado, que otorga también beneficios a la comunidad" (Abal de Hevia, 2016 p.15).

Es importante que lo anterior se distinga del asistencialismo que clásicamente se emplea como parte de metodologías pedagógicas de intervención dado el carácter protagónico de los actores en ambos extremos de la colaboración, haciendo protagonistas a los destinatarios con fin de ejercer una autonomía en el futuro. Abal de Hevia lo sintoniza de esta forma:
"La actividad comunitaria tal como se plantea en la propuesta del aprendizaje-servicio difiere de las prácticas asistenciales habituales basadas en la provisión de bienes o servicios, para en su lugar proponer practicas promocionales que exigen protagonismo de los destinatarios para que puedan desarrollar por sí mismos 
competencias y recursos que gradualmente estimulen la autonomía, e impacten en la calidad de vida. $Y$ este es un principio sustantivo que apunta a favorecer la transformación educativa y social" (Abal de Hevia, 2016 pp.19-20).

Una vez entregada una breve conceptualización del aprendizajeservicio, resulta interesante señalar alguna evidencia empírica, como la entregada por Gil-Gómez et al. (2016), quienes presentan los resultados de la aplicación de un programa de aprendizaje-servicio a profesores en formación en el ámbito de la Educación Física. En concreto se analiza su incidencia en el desarrollo de la competencia social y ciudadana, desde una perspectiva metodológica mixta. Las categorías de análisis de dicho estudio se presentan en la siguiente tabla:

Tabla 1. Dimensiones de la Competencia Social y Ciudadana

\begin{tabular}{|l|l|}
\hline DIMENSIONES & DEFINICIÓN \\
\hline $\begin{array}{l}\text { Conformidad } \\
\text { con lo } \\
\text { socialmente } \\
\text { correcto }\end{array}$ & $\begin{array}{l}\text { Respeto hacia las personas } \\
\text { con autoridad en el ámbito } \\
\text { académico, aceptación de las } \\
\text { normas de convivencia en } \\
\text { casa y respeto hacia las } \\
\text { cosas de los compañeros }\end{array}$ \\
\hline $\begin{array}{l}\text { Sensibilidad } \\
\text { social }\end{array}$ & $\begin{array}{l}\text { Empatía hacia otros, } \\
\text { capacidad de escuchar, } \\
\text { comprender y descubrir } \\
\text { cualidades en los demás, } \\
\text { satisfacción por compartir la } \\
\text { alegría cuando a otra persona } \\
\text { algo le sale bien y por estar } \\
\text { con los compañeros }\end{array}$ \\
\hline
\end{tabular}

\begin{tabular}{|c|c|}
\hline $\begin{array}{l}\text { Ayuda y } \\
\text { colaboración }\end{array}$ & $\begin{array}{l}\text { Generosidad con los demás, } \\
\text { capacidad de trabajar en } \\
\text { grupo y de ayudar a los } \\
\text { componentes de un equipo y } \\
\text { de dedicar tiempo a los } \\
\text { demás si lo necesitan }\end{array}$ \\
\hline $\begin{array}{l}\text { Seguridad y } \\
\text { firmeza en la } \\
\text { interacción }\end{array}$ & $\begin{array}{l}\text { Capacidad de reconocer } \\
\text { errores, defender derechos y } \\
\text { de dialogar para solucionarlo, } \\
\text { seguridad en uno mismo y } \\
\text { poder discutir cuando se } \\
\text { tenga la sensación de } \\
\text { injusticia de forma serena y } \\
\text { razonable }\end{array}$ \\
\hline $\begin{array}{l}\text { Liderazgo } \\
\text { prosocial }\end{array}$ & $\begin{array}{l}\text { Capacidad de movilizar a los } \\
\text { demás, de sugerir y aportar } \\
\text { ideas y la satisfacción por } \\
\text { organizar nuevas actividades }\end{array}$ \\
\hline $\begin{array}{l}\text { Responsabilidad } \\
\text { social }\end{array}$ & $\begin{array}{l}\text { Implicación personal para } \\
\text { actuar ante problemas } \\
\text { sociales aceptándolos como } \\
\text { parte de uno y aceptando la } \\
\text { responsabilidad individual, } \\
\text { capacidad de asumir } \\
\text { obligaciones sin que nadie lo } \\
\text { tenga que recordar y de } \\
\text { implicarse en proyectos para } \\
\text { mejorar la sociedad }\end{array}$ \\
\hline
\end{tabular}

Fuente: elaboración propia a partir de Gil-Gómez et al. (2016)

Finalmente reportan que el estudiantado que participa de aprendizaje-servicio muestra una mayor tendencia de mejora en dimensiones actitudinales del aprendizaje como conformidad con lo socialmente correcto, sensibilidad social, ayuda y colaboración y responsabilidad social, por lo que concluyen que es una buena herramienta para desarrollar su competencia social y ciudadana.

Peña, A. y Delgado, C. (2021). Usos del aprendizaje-servicio durante el proceso de formación de estudiantes en Educación Física: una revisión sistemática. RIDAS, Revista Iberoamericana de Aprendizaje Servicio, 11, 80-92. DOI10.1344/RIDAS2021.11.5 
Del mismo modo, Simsek (2020) con un grupo de 14 estudiantes de lengua extranjera (inglés) que participaron de un proyecto de aprendizaje-servicio, observó un cambio conceptual no sólo en sus perspectivas de enseñanza, del paradigma conductista al constructivista para la adquisición de conocimientos, sino también en sus enfoques de asociación comunitaria del patrón unidireccional al recíproco de altruismo hacia sus estudiantes. Además, informaron que habían desarrollado disposiciones eficaces en materia de enseñanza, aptitudes interpersonales y responsabilidad social, valoraron las recompensas intrínsecas por sus contribuciones al bienestar de los jóvenes y también apreciaron las oportunidades de aprendizaje profesional informal que ofrecía el proyecto en el que participaron. Dentro de sus conclusiones se refiere a cuatro recomendaciones a resaltar:

1. Añadir componentes de aprendizaje-servicio a los programas que ya existen, $y$ desarrolla una conexión única con el grupo del curso a trabajar.

2. Una planeación que incorpore el uso de una taxonomía de aprendizaje significativo y profundo, considerando instrucciones claras y explícitas del proceso.

3. Uso de enfoques mixtos de investigación al realizar estudios que soliciten conocer la eficiencia del proceso desde las voces de los protagonistas del servicio y de los actores involucrados del proyecto.

\section{Rills.}

4. Establecer una liga de universidades que colaboren en promover, compartir e investigar el Aprendizaje-Servicio, compartiendo las buenas prácticas y experticia, con fin a resolver los problemas locales de la comunidad (Simsek, 2020).

Lo anterior promueve puntos de contacto entre las emociones, la racionalidad de la persona en acción, el conocimiento teórico de la profesión movilizado con fines de aprendizaje profundo y significativo, brindando a la vez un espacio de desarrollo interpersonal $y$, al mismo tiempo de individuo participante en una sociedad democrática que tiende a lo justo.

Por otra parte, Escofet y Rubio (2019), en una investigación con 65 estudiantes universitarios participes de un proyecto de aprendizaje-servicio, registraron que estos valoran positivamente su participación, aunque señalan la necesidad de seguir mejorando el vínculo entre el aprendizaje y servicio. Asimismo, el estudio destaca la necesidad de profundizar y ampliar la práctica reflexiva como medio para fortalecer el vínculo formativo académico con el servicio realizado por los estudiantes. En su investigación se recalca que "no importando cuan motivante es la experiencia de aprendizaje-servicio en sí misma, perderá sentido y significado si no es acompañada continuamente por espacios y momentos de reflexión que supere nuestras emociones y avance hasta un posible aprendizaje" (Escofet y Rubio, 2019, p.168).

También es importante presentar que, 
como conclusiones del presente proyecto, la autopercepción del estudiantado es de un fortalecimiento de las competencias ligadas a la resolución de problemas, compromiso con la comunidad, dominios de contenido declarativo y trabajo en equipo. Por otra parte, las competencias que menor desarrollo tuvieron fueron búsqueda de información, conocimiento de distintas lenguas y conocimiento de herramientas de tecnología digital (Escofet y Rubio 2019), lo que nos previene de cuál es el foco de aprendizaje en un proyecto de esta naturaleza, anticipando remediales para la movilización de estas competencias.

A su vez, Goff et al. (2020) al finalizar el programa de intervenciones, recogieron de los estudiantes que en su mayoría buscaban extender su tiempo de servicio en la comunidad y/o aplicar el programa en otras comunidades y territorios por el valor de la experiencia de involucrarse en la actividad como estudiantes en formación profesional. Se relevan tres categorías de análisis a tener en cuenta en el presente reporte que fueron especialmente destacadas por los autores para entender el impacto de la experiencia de aprendizaje-servicio, y son: El Servicio como impulsor de la creación de un laboratorio de aprendizaje para el desarrollo profesional; Conciencia sobre los desafíos que enfrenta la juventud; y que el Servicio crea conexiones significativas (Goff et al., 2020)

Del mismo modo, Chin-Sook (2020) estudió la experiencia de 560 exestudiantes que participaron de proyectos de aprendizaje-servicio durante su formación de pregrado. En este estudio se identifican aspectos del aprendizaje-servicio que los estudiantes encuentran valorables. La siguiente tabla expone los aspectos y el total de respuestas de los aspectos identificados por los estudiantes como más valorables de aprendizaje-servicio.

Tabla 2. Aspectos identificados en el aprendizaje-servicio (Chin-Sook, 2020)

\begin{tabular}{|c|c|c|}
\hline \multicolumn{3}{|c|}{$\begin{array}{l}\text { Aspectos identificados en el } \\
\text { aprendizaje-servicio }\end{array}$} \\
\hline $\begin{array}{l}\text { A la pregunta } \\
\text { ¿Cuál fue la } \\
\text { experiencia que } \\
\text { MÁs disfrutaste } \\
\text { acerca de la } \\
\text { experiencia de } \\
\text { aprendizaje- } \\
\text { servicio? }\end{array}$ & \begin{tabular}{|l} 
Total de 55 \\
respuestas
\end{tabular} & $\begin{array}{l}\text { Promedio } \\
\text { por cada } \\
\text { respuesta }\end{array}$ \\
\hline $\begin{array}{l}\text { Formación de } \\
\text { relaciones y vínculos } \\
\text { con personas }\end{array}$ & 31 & 56,4 \\
\hline $\begin{array}{l}\text { Aplicación a } \\
\text { situaciones reales }\end{array}$ & 22 & 40 \\
\hline $\begin{array}{l}\text { Impacto significativo } \\
\text { del proyecto }\end{array}$ & 20 & 36,4 \\
\hline $\begin{array}{l}\text { Aprender sobre } \\
\text { desafíos de la } \\
\text { comunidad }\end{array}$ & 15 & 27,3 \\
\hline $\begin{array}{l}\text { Adquirir } \\
\text { herramientas, } \\
\text { incluyendo segunda } \\
\text { lengua }\end{array}$ & 8 & 14,5 \\
\hline Autonomía & 3 & 5,5 \\
\hline $\begin{array}{l}\text { Otras (ej. impacto } \\
\text { en la carrera, salir } \\
\text { de la zona de } \\
\text { confort) }\end{array}$ & 4 & 7,3 \\
\hline
\end{tabular}

Peña, A. y Delgado, C. (2021). Usos del aprendizaje-servicio durante el proceso de formación de estudiantes en Educación Física: una revisión sistemática. RIDAS, Revista Iberoamericana de Aprendizaje Servicio, 11, 80-92. DOI10.1344/RIDAS2021.11.5 


\begin{tabular}{|l|c|c|}
\hline $\begin{array}{l}\text { A la pregunta } \\
\text { ¿Qué aspectos } \\
\text { disfrutaste MENOS } \\
\text { de la experiencia } \\
\text { de aprendizaje- } \\
\text { servicio? }\end{array}$ & $\begin{array}{l}\text { Total de } \\
\text { respuestas } \\
\text { de } \mathbf{4 3}\end{array}$ & $\begin{array}{l}\text { Promedio } \\
\text { por cada } \\
\text { respuesta }\end{array}$ \\
\hline $\begin{array}{l}\text { Tiempo de } \\
\text { compromiso y } \\
\text { planificación }\end{array}$ & 9 & 20,9 \\
\hline $\begin{array}{l}\text { Salir de la zona de } \\
\text { confort }\end{array}$ & 8 & 18,6 \\
\hline $\begin{array}{l}\text { Desarrollar el } \\
\text { servicio que no se } \\
\text { prefería }\end{array}$ & 5 & 11,6 \\
\hline $\begin{array}{l}\text { Dificultades en el } \\
\text { trabajo de equipo }\end{array}$ & 5 & 11,6 \\
\hline $\begin{array}{l}\text { Falta de estructura o } \\
\text { falta de } \\
\text { entrenamiento } \\
\text { previo a las tareas }\end{array}$ & 3 & 7 \\
\hline $\begin{array}{l}\text { Diarios de reflexión } \\
\text { problemas y } \\
\text { malentendidos de } \\
\text { comunicación }\end{array}$ & 3 & \\
\hline $\begin{array}{l}\text { Otros (ej. } \\
\text { presentaciones } \\
\text { finales, viajes al } \\
\text { lugar, falta de } \\
\text { oportunidades de } \\
\text { continuidad) }\end{array}$ & 3 & \\
\hline
\end{tabular}

Fuente: elaboración propia

Los resultados muestran que la mayoría de los exalumnos reconocen su participación como una de las experiencias educativas más significativas en la universidad, que conducen a ganancias positivas en el conocimiento, las actitudes y comportamientos para interactuar con las comunidades. Los aspectos anteriormente levantados y sus resultados nos permiten mejorar las intenciones, planificación, los criterios y parámetros de evaluación la satisfacción de los estudiantes por participar en metodologías de aprendizaje-servicio, dado que dichos aspectos pueden dar una referencia para ser considerados en la elaboración, a futuro, de los modelos de evaluación de los proyectos pedagógicos que involucren aprendizaje-servicio.

El presente artículo realiza una descripción y análisis de la literatura relacionada con los usos del aprendizaje-servicio durante el proceso de formación de estudiantes en Educación Física, teniendo como fuente de exploración la base académica ERIC, durante el tiempo comprendido entre los años 2015 a 2020.

\section{Metodología}

El diseño, de corte exploratorio, se traduce operativamente en un plan de revisión sistemática, que contempla los criterios para la selección de los estudios, la estrategia de exploración y los métodos para la recolección y síntesis de la información.

\subsection{Búsqueda de información}

La búsqueda de artículos se realizó en la base de datos ERIC, utilizando las palabras claves 'Learning Service' y 'physical Education' y el booleano AND. Adicionalmente, se consideraron como criterios de inclusión investigaciones realizadas entre los años 2016-2020, artículos publicados en inglés, revisados por pares y llevados a cabo en el área de Educación Superior, excluyendo todas aquellas publicaciones que no 
contenían todos los criterios de inclusión antes mencionados.

\subsection{Depuración y aplicación de criterios de inclusión}

En cuanto al objetivo de la revisión sistemática, se seleccionaron los artículos que cumplieran en conjunto con dos requerimientos. En primer lugar, el artículo debía reportar hallazgos investigativos independientemente del abordaje metodológico usado. A su vez, el segundo requerimiento era que la investigación había contado con estudiantes universitarios en condición de participantes. Por lo anterior, artículos teóricos y de reflexión fueron excluidos.

\subsection{Sistematización de información}

Los documentos seleccionados para la revisión sistemática fueron estudiados y la siguiente información se extrajo a una matriz, registrando Autor/es, Año de Publicación, Muestra, Propósito de la investigación, Metodología y

Resultados.

\section{Resultados}

Un total de 774 artículos se identificaron como potenciales candidatos para la revisión. Luego de aplicar filtro por año de publicación, se encontraron 183 resultados de búsqueda. Posteriormente, se procedió a revisar los títulos de las publicaciones, descartando aquellas que no eran coherentes con los propósitos de esta investigación. De esta revisión, se registraron 8 publicaciones que contaban con títulos pertinentes con las variables investigadas. Por último, se realizó una lectura del resumen de los 8 artículos seleccionados, descartando 4 de ellos, que corresponden a artículos teóricos o de reflexión sobre la temática aprendizaje de servicio en el contexto de Educación Física (Fig. 1).

Figura 1. Diagrama PRISMA de búsqueda y selección de las fuentes analizadas

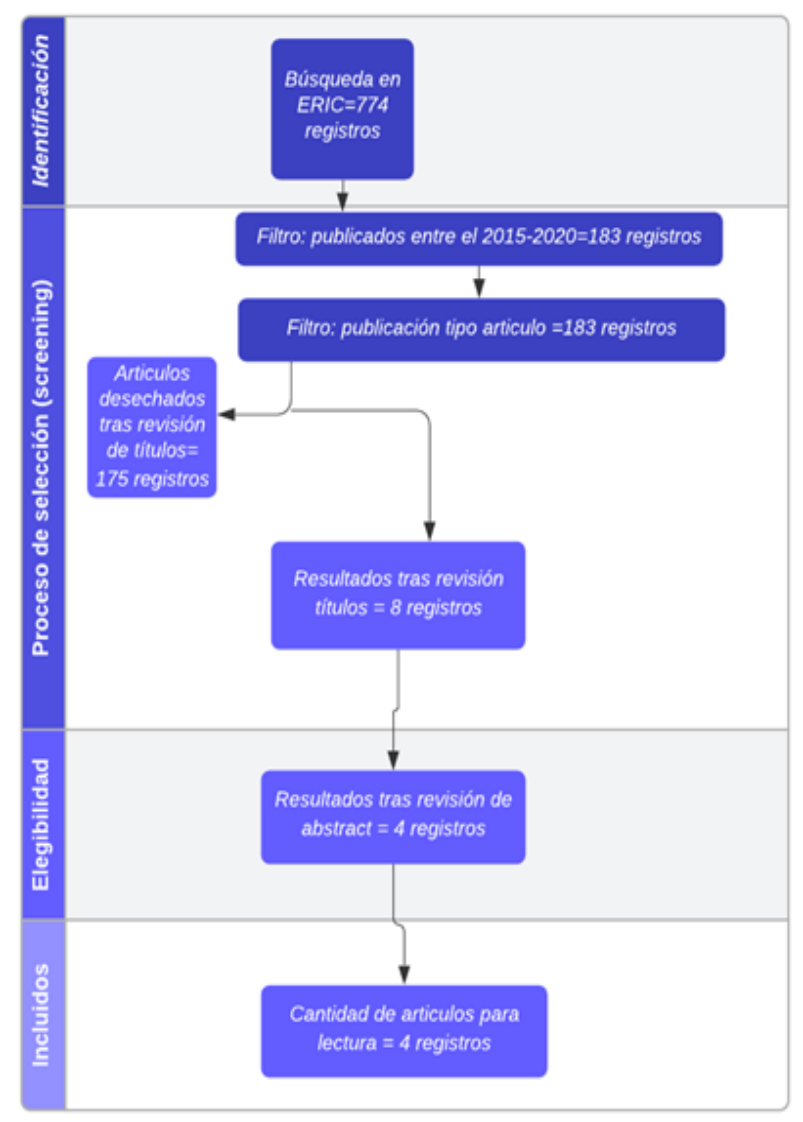

Fuente: elaboración propia

3.1 Año de publicación y cantidad de participantes

Sobre los años de publicación y cantidad de participantes en los artículos revisados, en la tabla 1 se 
sistematizan las investigaciones en función de estas variables. En ella, podemos observar que la investigación con mayor data y que aborda los usos que desde la Educación Física se dan al aprendizaje-servicio en Educación Superior, es la de Peralta et al. (2015) en que participaron 55 estudiantes. Luego, Ward et al. (2017) realizaron una investigación en que participaron 8 sujetos. Por último, los estudios más recientes son los realizados por ChivaBartoll et al. (2020) y Capella-Peris et al. (2020), con 169 y 96 participantes respectivamente.

A partir de lo anterior, observamos que dos de los cuatro artículos revisados fueron desarrollados durante el presente año. Al mismo tiempo, la mayor cantidad de participantes en las intervenciones se registró durante el año 2020.

Tabla 3. Sistematización de los artículos seleccionados en función del año de publicación y cantidad de participantes

\begin{tabular}{|c|c|c|}
\hline Autor/es & $\begin{array}{c}\text { Año de } \\
\text { publicación }\end{array}$ & Participantes \\
\hline $\begin{array}{c}\text { (Peralta et } \\
\text { al., 2015, p. } \\
\text { 261) }\end{array}$ & 2015 & 55 \\
\hline $\begin{array}{c}\text { (Ward et al., } \\
\text { 2017, p.76) }\end{array}$ & 2017 & 8 \\
\hline $\begin{array}{c}\text { (Chiva-Bartoll } \\
\text { et al., 2020, } \\
\text { p.397) }\end{array}$ & 2020 & 169 \\
\hline $\begin{array}{c}\text { (Capella-Peris } \\
\text { et al., 2020, } \\
\text { p.106) }\end{array}$ & 2020 & 96 \\
\hline
\end{tabular}

3.2 Abordajes metodológicos y propósitos de las investigaciones

Con respecto al abordaje metodológico y el propósito de los artículos revisados, en la tabla 2 se sistematizan las investigaciones en función de estas variables. Para ser más específicos, se observa que Capella-Peris et al. (2020) utilizaron un abordaje metodológico mixto y buscaron mediante un programa de aprendizaje-servicio, comparar el desarrollo de competencia docente ante dos modalidades diferentes de intervención dentro del mismo programa. Por otra parte, ChivaBartoll et al. (2020) y Peralta et al. (2015) desarrollaron investigaciones con una finalidad similar, puesto que buscaron analizar los efectos del aprendizaje-servicio sobre estudiantes de Educación Física. No obstante, estas investigaciones se diferencian en la metodología utilizada, puesto que en Chiva-Bartoll et al. (2020) utilizaron una metodología cualitativa, mientras que el grupo de Peralta et al. (2015) se orientó a la metodología mixta, siguiendo las recomendaciones ampliamente recomendadas. Del mismo modo, Ward et al. (2017) usaron una metodología cualitativa y tuvieron como propósito analizar los efectos de un programa de aprendizaje-servicio en función de la teoría del desequilibrio cognitivo.

Tabla 4. Sistematización de los artículos seleccionados en función del Propósito de la investigación y Abordaje metodológico

\begin{tabular}{|c|c|c|}
\hline Autor/es & $\begin{array}{c}\text { Propósito de } \\
\text { la } \\
\text { investigación }\end{array}$ & $\begin{array}{c}\text { Abordaje } \\
\text { metodológico }\end{array}$ \\
\hline
\end{tabular}

Fuente: elaboración propia

Peña, A. y Delgado, C. (2021). Usos del aprendizaje-servicio durante el proceso de formación de estudiantes en Educación Física: una revisión sistemática. RIDAS, Revista Iberoamericana de Aprendizaje Servicio, 11, 80-92. DOI10.1344/RIDAS2021.11.5 


\begin{tabular}{|c|c|c|}
\hline $\begin{array}{l}\text { (Capella- } \\
\text { Peris et } \\
\text { al., 2020, } \\
\text { p. 106) }\end{array}$ & $\begin{array}{l}\text { Comparar el } \\
\text { desarrollo de la } \\
\text { competencia } \\
\text { docente de los } \\
\text { profesores de } \\
\text { educación física } \\
\text { en pre-servicio } \\
\text { a través de dos } \\
\text { modalidades } \\
\text { diferentes de } \\
\text { intervención del } \\
\text { mismo programa } \\
\text { de aprendizaje- } \\
\text { servicio. }\end{array}$ & $\begin{array}{l}\text { Para desarrollar } \\
\text { la intervención } \\
\text { de aprendizaje- } \\
\text { servicio se } \\
\text { formaron } 2 \\
\text { grupos: grupo } \\
\text { experimental } 1 \\
\text { (EGI) y } \\
\text { experimental } 2 \\
\text { (EGII). El primer } \\
\text { grupo participó } \\
\text { en } 30 \text { sesiones } \\
\text { de } 1 \text { hora, } \\
\text { mientras que el } \\
\text { segundo grupo } \\
\text { sólo participó en } \\
9 . \text { El problema se } \\
\text { abordó utilizando } \\
\text { métodos mixtos } \\
\text { de triangulación. }\end{array}$ \\
\hline $\begin{array}{l}\text { (Chiva- } \\
\text { Bartoll et } \\
\text { al., 2020, } \\
\text { p. 397) }\end{array}$ & $\begin{array}{l}\text { El propósito de } \\
\text { este estudio fue } \\
\text { analizar el } \\
\text { impacto del } \\
\text { aprendizaje- } \\
\text { servicio en los } \\
\text { estudiantes de } \\
\text { educación física } \\
\text { (PETEs). }\end{array}$ & $\begin{array}{l}\text { El PETE completó } \\
\text { una de las } \\
\text { versiones de un } \\
\text { programa de } \\
\text { aprendizaje- } \\
\text { servicio llevado a } \\
\text { cabo entre 2015- } \\
2018 \text {. Este } \\
\text { programa } \\
\text { consistió en } \\
\text { diseñar y dirigir } \\
\text { sesiones de } \\
\text { juegos de motor } \\
\text { para facilitar la } \\
\text { inclusión de } 116 \\
\text { niños con } \\
\text { necesidades } \\
\text { educativas } \\
\text { especiales (SEN). } \\
\text { Se utilizaron } \\
\text { diarios de } \\
\text { reflexión como } \\
\text { instrumento para } \\
\text { recoger } \\
\text { información de } \\
\text { sus experiencias. }\end{array}$ \\
\hline $\begin{array}{l}\text { (Peralta } \\
\text { et al., } \\
2015, \text { p. } \\
261 \text { ) }\end{array}$ & $\begin{array}{l}\text { En este trabajo, } \\
\text { investigamos los } \\
\text { efectos de un } \\
\text { programa de } \\
\text { experiencia de } \\
\text { aprendizaje- } \\
\text { servicio sobre los } \\
\text { profesores de } \\
\text { educación física } \\
\text { previos al } \\
\text { servicio. }\end{array}$ & $\begin{array}{l}\text { Basado en los } \\
\text { principios } \\
\text { teóricos de las } \\
\text { metodologías de } \\
\text { investigación } \\
\text { indígenas, el } \\
\text { aprendizaje } \\
\text { experimental y la } \\
\text { reflexión crítica, } \\
\text { examinamos a } \\
55 \text { estudiantes } \\
\text { de último año de } \\
\text { educación física }\end{array}$ \\
\hline
\end{tabular}

\begin{tabular}{|c|c|c|}
\hline & & $\begin{array}{l}\text { previa al servicio } \\
\text { como maestros. } \\
\text { Medidas de sus } \\
\text { experiencias } \\
\text { incluye revistas } \\
\text { de reflexión, } \\
\text { escalas de } \\
\text { competencia de } \\
\text { enseñanza } \\
\text { multicultural y } \\
\text { entrevistas de } \\
\text { grupos de } \\
\text { discusión. }\end{array}$ \\
\hline $\begin{array}{l}\text { (Ward et } \\
\text { al., 2017, } \\
\text { p. 76) }\end{array}$ & $\begin{array}{l}\text { El propósito de } \\
\text { este estudio fue } \\
\text { explorar las } \\
\text { experiencias de } \\
\text { los maestros } \\
\text { antes del servicio } \\
\text { con la teoría del } \\
\text { desequilibrio } \\
\text { cognitivo (DC) } \\
\text { durante un } \\
\text { proyecto de } \\
\text { aprendizaje- } \\
\text { servicio en una } \\
\text { experiencia de } \\
\text { estudio en el } \\
\text { extranjero }\end{array}$ & $\begin{array}{l}\text { Se utilizó un } \\
\text { estudio de caso } \\
\text { con } 8 \\
\text { participantes. Las } \\
\text { fuentes de datos } \\
\text { fueron } \\
\text { entrevistas } \\
\text { formales, vídeos } \\
\text { de planificación, } \\
\text { vídeos de } \\
\text { enseñanza, } \\
\text { vídeos de } \\
\text { sesiones de } \\
\text { reflexión y } \\
\text { entrevistas } \\
\text { informales. }\end{array}$ \\
\hline
\end{tabular}

Fuente: elaboración propia

\subsection{Resultados obtenidos}

En relación con los resultados obtenidos, en la tabla 3 se sistematizan las investigaciones en función de esta variable. Es necesario señalar, que los dos grupos que participaron del estudio de Capella-Peris et al. (2020) lograron a través del programa de aprendizajeservicio mejorar significativamente la competencia docente, sin encontrar diferencias en las competencias docentes al comparar ambos grupos. Dicha mejora, se complementó con reflexiones realizadas por los estudiantes luego de finalizar el programa de intervención. Del mismo modo, Chiva-Bartoll et al. (2020) 


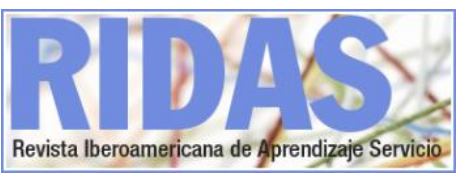

reportaron que los estudiantes de Educación Física participes del proyecto de aprendizaje-servicio, lograron desarrollar una experiencia educativa inclusiva, vinculando la teoría y la práctica de una manera verdaderamente operativa. Asimismo, Peralta et al. (2015) reportaron una mejora significativa en el ámbito de competencias docentes y dieron cuenta de la capacidad del estudiantado para desafiar prejuicios respecto a estudiantes indígenas durante la intervención de aprendizaje-servicio realizada, movilizando ámbitos del sentido profesional. Por otra parte, Ward et al. (2017) registraron la información proporcionada por los participantes en cuatro dimensiones, relacionadas con procesos de transformación respecto a su rol como futuros docentes y se describe a continuación:

Tabla 5. Sistematización de los artículos seleccionados en función de los Resultados obtenidos

\begin{tabular}{|c|c|}
\hline Autor/es & Resultados \\
\hline $\begin{array}{l}\text { (Capella- } \\
\text { Peris et } \\
\text { al., } \\
2020, p . \\
106 \text { ) }\end{array}$ & $\begin{array}{l}\text { Al realizar una comparación pre-post test } \\
\text { sobre competencias docentes, los valores } \\
\text { obtenidos al aplicar la prueba t para } \\
\text { muestras emparejadas fue de } t(39)= \\
5.618,(p .01) \text { para el EGI y t ( } 39)= \\
<5.905,(p .01) \text { para el EGII. Por lo tanto, } \\
\text { hubo diferencias significativas entre las } \\
\text { medidas anteriores y posteriores a la } \\
\text { prueba en ambos casos. Por otro lado, en } \\
\text { la interpretación cualitativa se } \\
\text { complementaba esta opinión, } \\
\text { reflexionando sobre cómo se desarrolló } \\
\text { este aprendizaje. }\end{array}$ \\
\hline $\begin{array}{l}\text { (Chiva- } \\
\text { Bartoll } \\
\text { et al., } \\
2020, p . \\
397 \text { ) }\end{array}$ & $\begin{array}{l}\text { El aprendizaje-servicio proporcionó a los } \\
\text { profesores de pregrado una experiencia } \\
\text { educativa inclusiva, permitiéndoles } \\
\text { vincular la teoría y la práctica de una } \\
\text { manera verdaderamente operativa. }\end{array}$ \\
\hline
\end{tabular}

\begin{tabular}{|c|c|}
\hline $\begin{array}{l}\text { (Peralta } \\
\text { et al., } \\
2015, \text { p. } \\
261 \text { ) }\end{array}$ & $\begin{array}{l}\text { Los resultados muestran cambios } \\
\text { significativos en las percepciones de los } \\
\text { profesores antes del servicio en el ambito } \\
\text { de competencia ( } p<0,001 \text { ). Los } \\
\text { maestros de pre-servicio fueron capaces } \\
\text { de desafiar sus prejuicios sobre los } \\
\text { estudiantes indígenas, planear y ejecutar } \\
\text { los planes centrados en los estudiantes y } \\
\text { pedagogías culturalmente relevantes. }\end{array}$ \\
\hline $\begin{array}{l}\text { (Ward et } \\
\text { al., } \\
2017, \text { p. } \\
76)\end{array}$ & $\begin{array}{l}\text { Los resultados indicaron cuatro temas: 'Lo } \\
\text { hicimos nuestro', 'Campamento de verano } \\
\text { para profesores', 'Lucha y desequilibrio', y } \\
\text { 'Al final fue una transformación': } \\
\text { Lo hicimos nuestro: Describieron esto } \\
\text { como tener el control tanto en términos } \\
\text { de lo que podían aprender como de lo que } \\
\text { podían enseñar. } \\
\text { Campamento de verano para profesores: } \\
\text { Esta experiencia se resumió en la frase, } \\
\text { "Es como un campamento de verano para } \\
\text { profesores" (Bert), en que como los } \\
\text { campamentos de verano para jóvenes, } \\
\text { esta corta experiencia rápidamente se } \\
\text { volvió intensamente poderosa. Esto fue } \\
\text { tanto positivo como negativo. Les } \\
\text { permitió adaptarse, pero también les } \\
\text { obligó a unirse. } \\
\text { Lucha y desequilibrio: Este tema se } \\
\text { describe en términos del momento en que } \\
\text { los PST encontraron una oposición que los } \\
\text { hizo detenerse y reflexionar sobre su } \\
\text { enseñanza. } \\
\text { Al final fue una transformación: Esta } \\
\text { transformación fue el resultado de la } \\
\text { resolución positiva de este desequilibrio } \\
\text { logrado sólo después de luchar o trabajar } \\
\text { en algo que era difícil o inesperado } \\
\text { (Farmer } 2010 ; \text { Walton, } 2011, \text { como se citó } \\
\text { en Ward et al., } 2017, \text { p.76). }\end{array}$ \\
\hline
\end{tabular}

Fuente: elaboración propia

\section{Discusión}

Una vez finalizado el proceso de análisis de los artículos seleccionados para esta revisión, resulta pertinente mencionar algunas ideas relevantes. En concreto,

Peña, A. y Delgado, C. (2021). Usos del aprendizaje-servicio durante el proceso de formación de estudiantes en Educación Física: una revisión sistemática. RIDAS, Revista Iberoamericana de Aprendizaje Servicio, 11, 80-92. DOI10.1344/RIDAS2021.11.5 
se debe mencionar el escaso número de publicaciones encontradas en que se relaciona el aprendizaje-servicio con estudiantes de pregrado de la carrera de Educación Física. No obstante, pese al escaso número de investigaciones, los resultados obtenidos en cada una de ellas dan cuenta de una gran relevancia e impacto positivo en los estudiantes de Educación Física que participan de intervenciones basadas en aprendizajeservicio y la proyección en su desarrollo profesional.

Con respecto a los usos que desde la Educación Física se ha dado al aprendizaje-servicio, se ha logrado identificar dos grandes usos. El primero de ellos, relacionado con el uso del aprendizaje-servicio como una instancia para desarrollar o mejorar competencias docentes de cara al ejercicio profesional que tendrá lugar al finalizar la formación de pregrado. Este tipo de enfoque fue observado en las investigaciones de Capella-Peris et al. (2020) y Chiva-Bartoll et al. (2020). Por otra parte, el segundo uso relacionado con el aprendizaje-servicio, concibe a este como una metodología para desarrollar o abordar conceptos o ideas puntuales y posteriormente analizar el ejercicio teórico-práctico en función de la variable en cuestión. Ejemplos de lo anteriormente dicho se observan en los estudios de Peralta et al. (2015), quienes utilizaron el aprendizaje-servicio para abordar prejuicios respecto a la población indígena. Asimismo, Ward et al. (2017) dieron uso del aprendizaje-servicio para llevar a la práctica la teoría del desequilibrio cognitivo (DC).

\section{Conclusión}

Para finalizar, la respuesta a la pregunta inicial es: si, es pertinente formar a los profesores de educación física con experiencias de aprendizaje-servicio. Y, es necesario destacar dos grandes ideas que surgen del proceso de revisión realizado. La primera de ellas, es que la literatura actual cuenta con un reducido número de publicaciones en que se relacione el aprendizajeservicio con estudiantes de pregrado de Educación Física, razón por la cual resulta necesario ampliar a otras fuentes de repositorios para continuar revisando en una mayor cantidad de investigaciones sobre esta temática, el impacto en la consecución de una formación profesional integral con el fin de esclarecer el potencial educativo de esta metodología de aprendizaje. Por otra parte, y a pesar de la baja cantidad de artículos, la segunda idea es referida al impacto positivo que han reportado las publicaciones encontradas sobre este tema, y que entrega indicios de que la metodología de aprendizajeservicio es una experiencia de aprendizaje de suma importancia para el estudiantado de pre grado, en general, y que podría la realización de estas experiencias en la formación profesional puede generar cambios positivos en su posterior desempeño profesional, dado los aspectos de compromiso social y responsabilidad social ciudadana. En este sentido, a partir de la revisión realizada, también se sugiere una mayor utilización de esta metodología de aprendizaje durante el desarrollo de los niveles de pregrado de la carrera de Educación Física, independientemente a si dicha

\footnotetext{
Peña, A. y Delgado, C. (2021). Usos del aprendizaje-servicio durante el proceso de formación de estudiantes en Educación Física: una revisión sistemática. RIDAS, Revista Iberoamericana de Aprendizaje Servicio, 11, 80-92. DOI10.1344/RIDAS2021.11.5
} 
intervención será reportada en forma de artículo investigativo.

\section{Referencias bibliográficas}

Abal de Hevia, I. (2016). Aprendizaje Servicio solidario: una propuesta pedagógica innovadora. RIDAS, Revista Iberoamericana de Aprendizaje y Servicio, (2), 3-32. doi: 10.1344/RIDAS2016.2.2

Capella-Peris, C., Gil-Gómez, J., \& Chiva-Bartoll, Ò. (2020). Innovative Analysis of Service-Learning Effects in Physical Education: A Mixed-Methods Approach. Journal of Teaching in Physical Education, 39(1), 102-110. doi: $10.1123 /$ jtpe.2019-0030

Chin-Sook, P. (2020). Exploring the Long-term Impact of Service-Learning: Former Students of Spanish Revisit their Community Engagement Experiences. Hispania, 103(1), 67-85.

Chiva-Bartoll, O., Capella-Peris, C., \& Salvador-García, C. (2020). ServiceLearning in physical education teacher education: towards a critical and inclusive perspective. Journal of Education for Teaching, 46(3),1-13. doi: $10.1080 / 02607476.2020 .1733400$

Escofet, A., \& Rubio, L. (2019). Impact Analysis of a Service-Learning University Program From the Student Perspective. Journal of Higher Education Outreach and Engagement, 23(3), 159174. Recuperado de https://openjournals.libs.uga.edu/jheoe Larticle/view/1526/1463

Gil-Gómez, J., Moliner-García, O., y Chiva-Bartoll, Ó. (2016). Una experiencia de aprendizaje-servicio en futuros docentes: desarrollo de la competencia social y ciudadana. Revista Complutense de Educación, 27(1) 5373. doi: 10.5209/rev_RCED.2016.v27.n1.45071

Goff, J., Hill, E., Eckhoff, A. \& Dice, T. (2020). Examining the High-impact Practice of Service-learning: Written Reflections of Undergraduate Recreation Majors. SCHOLE: A Journal of Leisure Studies and Recreation Education, 35(1), 1-14. doi:

10.1080/1937156X.2020.1720444

Peralta, L., O'Connor, D., Cotton, W. \& Bennie, A. (2015). Pre-service physical education teachers' Indigenous knowledge, cultural competency and pedagogy: a service learning intervention. Teaching Education, 27(3), 248-266. doi: $10.1080 / 10476210.2015 .1113248$

Simsek, M. (2020). The impact of service-learning on EFL teacher candidates' academic and personal development. European Journal of Educational Research, 9(1), 1-17. doi: 10.12973/eu-jer.9.1.1

Ward, S., Pellett, H. H., \& Perez, M. I. (2017). Cognitive Disequilibrium and Service-Learning in Physical Education Teacher Education: Perceptions of PreService Teachers in a Study Abroad Experience. Journal of Teaching in Physical Education, 36(1), 70-82. doi: 10.1123/jtpe.2015-0006

\footnotetext{
Peña, A. y Delgado, C. (2021). Usos del aprendizaje-servicio durante el proceso de formación de estudiantes en Educación Física: una revisión sistemática. RIDAS, Revista Iberoamericana de Aprendizaje Servicio, 11, 80-92. DOI10.1344/RIDAS2021.11.5
} 